\title{
Survival after Parathyroidectomy in Patients with End-stage Renal Disease and Severe Hyperparathyroidism
}

\author{
Andrea Trombetti, MD, ${ }^{1}$ Catherine Stoermann, MD, ${ }^{2}$ John H. Robert, MD, ${ }^{3}$ \\ François R. Herrmann, MD, ${ }^{1}$ Pietra Pennisi, MD, ${ }^{1}$ Pierre-Yves Martin, MD, ${ }^{2}$ \\ René Rizzoli, MD ${ }^{1}$
}

${ }^{1}$ Service of Bone Diseases (WHO Collaborating Center for Osteoporosis Prevention), Department of Rehabilitation and Geriatrics, University Hospital of Geneva, 1211 Geneva 14, Switzerland

${ }^{2}$ Service of Nephrology, Department of Internal Medicine, University Hospital of Geneva, 1211 Geneva 14, Switzerland

${ }^{3}$ Thoracic Surgery Unit, Department of Surgery, University Hospital of Geneva, 1211 Geneva 14, Switzerland

\begin{abstract}
Background: Patients with end-stage renal disease (ESRD) and secondary hyperparathyroidism (SHPT) are at high risk of mortality. Whether an increased risk of death persists after a parathyroidectomy (PTX) is not clearly established.

Subjects and methods: The survival of 40 patients with ESRD and SHPT who underwent PTX was compared with that of 664 ESRD patients.

Results: From first dialysis, a lower mortality rate was found in the group of patients who underwent PTX than in the nonoperated ESRD group (hazard ratio: 0.23 ; 95\% Cl: $0.14-0.37$ ). The patients who underwent PTX were younger, had a longer time on dialysis, and had a higher prevalence of kidney transplantation. The mean number of comorbidities was lower (Charlson score $4.2 \pm 2.1$ versus $6.4 \pm 2.9, p<0.001)$. Then, we randomly selected two matched controls for each PTX case (80 controls, 40 PTX) who had at least an equivalent mean duration of dialysis between the first dialysis and PTX of the PTX group. In a univariate model, there was a trend for PTX being associated with prolonged survival. The mortality was higher both among those at an advanced age and those with a high Charlson score. Adjustments for these covariates made the effect of PTX no more significant.

Conclusions: The risk of death of patients with severe SHPT leading to PTX differed from that of nonoperated subjects. The apparent differences in survival may be related to the number and severity of associated comorbidities. ESRD patients who undergo PTX may represent a subset of healthier subjects.
\end{abstract}

S econdary hyperparathyroidism (SHPT) is a common complication of chronic kidney disease (CKD) that can lead to clinically significant bone disease.$^{1,2}$ It is also associated with soft-tissue and vascular calcifications,

Correspondence to: Andrea Trombetti, MD, e-mail: Andrea.Trombetti@hcuge.ch cardiovascular disease, and calcifying uremic arteriopathy, all of which may contribute to increased risk of cardiovascular morbidity and mortality among CKD patients, ${ }^{3-9}$ with a relative risk of death between 1.06 and 3.9.

Secondary hyperparathyroidism arises from disturbances in calcium, phosphorus, vitamin $\mathrm{D}$, and parathyroid hormone metabolism, which develop at an early 
stage of $C K D^{10}$ and become more prominent as kidney function declines. To overcome deleterious effects of SHPT, standard therapies recommended to correct mineral metabolism and bone disease include calcium supplementation, dietary phosphorus restriction, phosphatebinding agents, and/or vitamin D metabolites. ${ }^{1,11}$ However, these agents frequently alter the serum levels of calcium and phosphorus, which result in worsening of serum values and significant extraskeletal morbidity and mortality. Calcimimetics, a new class of drugs offer the possibility to selectively reduce the levels of PTH, serum calcium, and phosphate at the same time. However, whether these effects can contribute to improved survival remains to be demonstrated. ${ }^{1,12}$ Thus, PTX in patients on dialysis or in those who have had renal transplants is still frequently required for the treatment of severe SHPT, although recent studies have shown that the percentage of dialysis patients undergoing subtotal or total PTX is declining, ${ }^{13,14}$ possibly as a result of more effective medical management.

Whether PTX modifies the mortality rate among patients with end-stage renal disease (ESRD) and severe HPT was studied recently in an observational matched cohort study using data from the United States Renal Database System (USRDS). It was shown that PTX patients displayed prolonged survival during chronic dialysis, with a $15 \%$ reduced risk of death. However, an important limitation of that study is that patient characteristics, in particular comorbidities and cardiovascular risk factors, were not taken into account in calculating the risk of death. Indeed, this may potentially explain the differences in mortality rates. ${ }^{15}$ The present study was undertaken to determine whether patients operated on for SHPT would be at lower risk of premature death, and to examine whether differences persisted when all comorbidities were considered. We thus analyzed survival after PTX in a well-defined and closely followed cohort of patients in whom cardiovascular risk factors and comorbidities could be carefully recorded and analyzed.

\section{SUBJECTS AND METHODS}

Between 1977 and 2002, 741 patients were treated in our institution for end-stage renal disease. The following patients were excluded from the analysis: 19 with parathyroidectomy following a successful kidney transplant (tertiary hyperparathyroidism), 13 patients with 3 or more kidney transplants ( 9 with a history of parathyroidectomy and 4 never operated), and 5 whose date of first dialysis was not known.
A total of 40 patients (18 males, 22 females) with ESRD and severe hyperparathyroidism underwent PTX between 1977 and 2002 at the Geneva University Hospital, a large acute-care teaching hospital that manages $95 \%$ of cases for Geneva and its environs, representing a population of around 400,000 . The survival rates of those 40 patients were compared to those of 664 ESRD patients treated at the same institution during the same period.

Renal failure was due to chronic glomerulonephritis $(n=13)$, polycystic kidney disease $(n=9)$, tubulointerstitial nephropathy $(n=12)$, vascular disease $(n=4)$, diabetes $(n=1)$, or undetermined causes $(n=1)$. Seventy-three percent $(n=29)$ were treated with hemodialysis, $10 \%(n=4)$ with continuous ambulatory peritoneal dialysis, and $18 \%(n=7)$ with the two treatments consecutively. This proportional distribution was quite similar to that of the control group. Mean age at surgery was $47.7 \pm 14.7$ years (mean \pm SD, range: $20-74$ years) and the mean time between the first dialysis and PTX was 5.3 years $(95 \% \mathrm{Cl}: 0.2-17.5)$. Initial PTX involved removal of 1 to 3 parathyroid glands ( $n=12,32 \%)$; subtotal PTX $(n=21,57 \%)$, or total PTX with autotransplantation $(n=4,10 \%)$. Information was not available for 3 patients. Surgery was undertaken in 1 patient just before the onset of dialysis; in the other 39 (96\%), during the period of dialysis. Age at first dialysis, gender, and year and date of surgery were noted for each patient. If the patient died during the study period, the year and date of death (but not the main cause of death) were recorded. For each patient, we retrospectively assessed the number of cardiovascular disease risk factors. Dyslipidemia, diabetes and impaired glucose tolerance, hypertension, tobacco use at any time during the follow-up, obesity, and family history of cardiovascular disease before the age of 65 years were taken into account. Comorbid conditions, present at the start of dialysis or that developed during the follow-up were obtained from the patient records. A modified Charlson's Comorbidity Index was computed for each patient. ${ }^{16}$ Patients were considered to have a comorbid condition if a listed disorder (Table 1) was mentioned in his/her medical records, or if the patient had been treated for it. Four comorbidity groups were established: low comorbidity (scores $\leq 3$ ), moderate comorbidity (scores 4 and 5), high comorbidity (scores 6 and 7 ), and very high comorbidity (scores $\geq 8$ ).

\section{Statistical analysis}

Summary results are given as the mean \pm standard deviation. When the data were not normally distributed, the summary descriptive statistics were the median and 
Table 1.

Modified Charlson Comorbidity Index

\begin{tabular}{cl}
\hline Comorbidity score $^{1}$ & \multicolumn{1}{c}{ Condition } \\
\hline 1 & Coronary artery \\
& disease \\
& Congestive heart failure \\
& Peripheral vascular disease \\
& Cerebrovascular disease \\
& Dementia \\
& Chronic pulmonary disease \\
& Connective tissue disorder \\
& Peptic ulcer disease \\
& Mild liver disease \\
& Diabetes \\
& Hemiplegia \\
& Moderate or severe renal disease \\
& Diabetes with end-organ damage \\
& Any tumor, leukemia, lymphoma \\
& Moderate or severe \\
& liver disease \\
& Metastatic solid tumor \\
& AlDs
\end{tabular}

${ }^{1}$ In addition, for each decade $>40$ years of age, a score of 1 was added to the comorbidity score.

${ }^{2}$ Nondiabetic patients received a minimum score of 2 for moderate to severe renal disease, and diabetic patients received a minimum of 4 ( 2 for diabetic end-organ damage and 2 for end-stage renal disease)

interquartile range. Comparisons of continuous variables between two or more groups were performed with Student's $t$-test or analysis of variance (ANOVA). When appropriate, a non-parametric Kruskal-Wallis test was applied. Chi-square tests were used for comparing proportions. The survival of PTX patients and controls following the first dialysis was quantified using survival function estimates based on the Kaplan-Meier method. ${ }^{17}$ Follow-up started on the date of first dialysis and continued until death or censoring. Censoring events were survival on 1 March 2003 or at the time of the first successful kidney transplant. A kidney transplant was judged successful if survival of the transplant was longer than 1 year. The resulting observed survival curves were compared using a log-rank test. ${ }^{18}$ Because of the large age discrepancy between PTX patients and non-PTX patients and their relatively small number, it was not possible to match each PTX case concomitantly for age and duration of dialysis, or for comorbid conditions. As a result of a large variation in the duration of dialysis, we randomly selected two controls for each PTX case who had at least an equivalent mean duration of dialysis ( \pm 2 years), between the first dialysis and PTX, in order to analyze the effect of PTX on survival.
A conditional logistic regression was used to estimate the influence of patient characteristics on the mortality rate. Independent variables evaluated were gender, age at first dialysis, number of cardiovascular risk factors, year when dialysis was started ( \pm 10 years), modality of dialysis (hemodialysis, peritoneal dialysis, or both, at any time during the follow-up), modified Charlson score, and parathyroidectomy.

The significance level for two-sided $p$ values was 0.05 in all tests. The data were analyzed using the STATA statistical software package (version 9.2; Stata Corporation, College Station, TX).

\section{RESULTS}

\section{Patients characteristics}

At the time of PTX, $40 \%$ of the patients had already experienced one or more cardiovascular events (coronary artery disease with angina pectoris or myocardial infarction: $7 / 40$ (18\%); cardiac insufficiency: 7/40 (18\%); acute stroke: $3 / 40(8 \%)$; or cardiac arrhythmia disorders: $2 / 40(5 \%))$. About half the patients $(58 \%)$ had symptomatic SHPT, with diffuse bone or joint pains $(17 / 40,43 \%)$, general symptoms (fatigue, muscle weakness, anorexia, $3 / 40,8 \%)$, neuropsychological disorders $(1 / 40,3 \%)$, and/ or pruritus $(2 / 40,5 \%)$. Three patients $(8 \%)$ had experienced a low trauma fracture and $10(25 \%)$ were clearly identified by dual-energy $\mathrm{x}$-ray absorptiometry as having osteoporosis. Osteitis fibrosa was diagnosed based on radiologic examination of 11 patients. The main cause of surgical referral was refractory hypercalcemia $(n=29$; $73 \%$ patients) or hyperphosphatemia $(n=23 ; 58 \%$ of the patients). Data on serum PTH levels were available for 28 patients before surgery. The mean serum PTH level was $19 \pm 15$ times the upper limit of the normal range.

The patients who underwent parathyroid surgery for secondary hyperparathyroidism had different characteristics from those of the control group (Table 2). The primary causes of end-stage renal failure were different. The most significant differences were younger age at first dialysis, a longer period of dialysis, and a higher prevalence of kidney transplantation, even with unsuccessful outcome, but a lower mean number of comorbidities. Cardiovascular risk factors and dialysis modalities did not differ between the two groups.

\section{Survival rate}

Only one PTX patient died within one year of surgery. Using survival function estimates based on the Kaplan- 
Table 2.

Patients' characteristics for the whole population

\begin{tabular}{|c|c|c|c|c|c|c|c|c|c|}
\hline & \multicolumn{4}{|c|}{$\begin{array}{l}\text { Dialyzed patients without PTX } \\
\qquad(n=664)\end{array}$} & \multicolumn{4}{|c|}{$\begin{array}{l}\text { Dialyzed patients with PTX } \\
\qquad(n=40)\end{array}$} & \multirow[t]{2}{*}{$P$} \\
\hline & $\mathrm{N}(\%)$ & Mean $\pm S D$ & $\min$ & $\max$ & $\mathrm{N}(\%)$ & Mean \pm SD & $\min$ & $\max$ & \\
\hline Gender $\mathrm{N}$ (\% of male) & $391(59)$ & & & & $18(45)$ & & & & 0.060 \\
\hline \multicolumn{10}{|l|}{ Cause of renal failure } \\
\hline Chronic glomerulonephritis & $159(24)$ & & & & $13(33)$ & & & & 0.151 \\
\hline Polycystic kidney disease & $62(9)$ & & & & $9(23)$ & & & & 0.014 \\
\hline Vascular & $199(30)$ & & & & $4(10)$ & & & & 0.001 \\
\hline Tubulointerstitial & $117(18)$ & & & & $12(30)$ & & & & 0.045 \\
\hline Diabetes & $113(17)$ & & & & $1(3)$ & & & & 0.001 \\
\hline Other or unknown & $74(11)$ & & & & $1(3)$ & & & & 0.059 \\
\hline Age at first dialysis & & $57.2 \pm 17.1$ & 11 & 90 & & $42.6 \pm 15.2$ & 14 & 69 & 0.001 \\
\hline Modality of dialysis & & & & & & & & & 0.708 \\
\hline Hemodialysis & $509(77)$ & & & & $29(73)$ & & & & \\
\hline Peritoneal dialysis & $52(8)$ & & & & $4(10)$ & & & & \\
\hline Both modalities & $103(16)$ & & & & $7(18)$ & & & & \\
\hline Time on dialysis (years) & & $2.9 \pm 3.3$ & 0 & 28 & & $10.2 \pm 7.2$ & 0 & 28 & 0.001 \\
\hline Renal graft $(\%)$ & $252(38)$ & & & & $22(55)$ & & & & 0.025 \\
\hline Number of renal transplantations (RT) & & & & & & & & & 0.005 \\
\hline Never & $411(62)$ & & & & $18(45)$ & & & & \\
\hline One RT & $221(33)$ & & & & $15(38)$ & & & & \\
\hline Two RT & $32(5)$ & & & & $7(18)$ & & & & \\
\hline Functioning renal graft (\%) & $213(32)$ & & & & $15(38)$ & & & & 0.291 \\
\hline Mean number of cardiovascular risk factors & & $1.4 \pm 0.9$ & 0 & 3 & & $1.4 \pm 1.0$ & 0 & 3 & 0.882 \\
\hline Mean modified Charlson score & & $6.4 \pm 2.9$ & 2 & 17 & & $4.2 \pm 2.1$ & 2 & 17 & 0.001 \\
\hline \multicolumn{10}{|l|}{ Charlson score } \\
\hline Low score $(\leq 3)$ & $123(19)$ & & & & $18(45)$ & & & & \\
\hline Moderate score (4 or 5$)$ & $143(22)$ & & & & $13(33)$ & & & & \\
\hline High score $(6$ or 7$)$ & $167(25)$ & & & & $7(18)$ & & & & \\
\hline Very high score $(\geq 8)$ & $231(35)$ & & & & $2(5)$ & & & & 0.001 \\
\hline
\end{tabular}

Meier method, it was found that the mortality rate after the first dialysis was significantly lower in the PTX group (hazard ratio: $0.23 ; 95 \% \mathrm{Cl}: 0.14-0.37$ ) (Fig. 1). Because of the differences between the two populations (Table 2), we tried specifically to evaluate the influence of PTX on survival. We randomly selected two controls for each PTX case, who had at least an equivalent mean duration of dialysis, between the first dialysis and PTX for the PTX group and the beginning of dialysis and any censoring event in the control group ( \pm 2 years) to analyze the effect of PTX on survival (40 PTX patients and 80 controls) Some differences were still present after this matching: younger age at time of first dialysis, a lower number of comorbidities, and a higher number of renal transplantations (Table 3). The censoring at time of renal transplantation alleviated the confounding effect of transplantation on survival. Then, conditional logistic regression was used to estimate the influence of patient characteristics on the mortality rate. Independent variables evaluated were gender, age at first dialysis, number of cardiovascular risk factors, year of initiation of dialysis

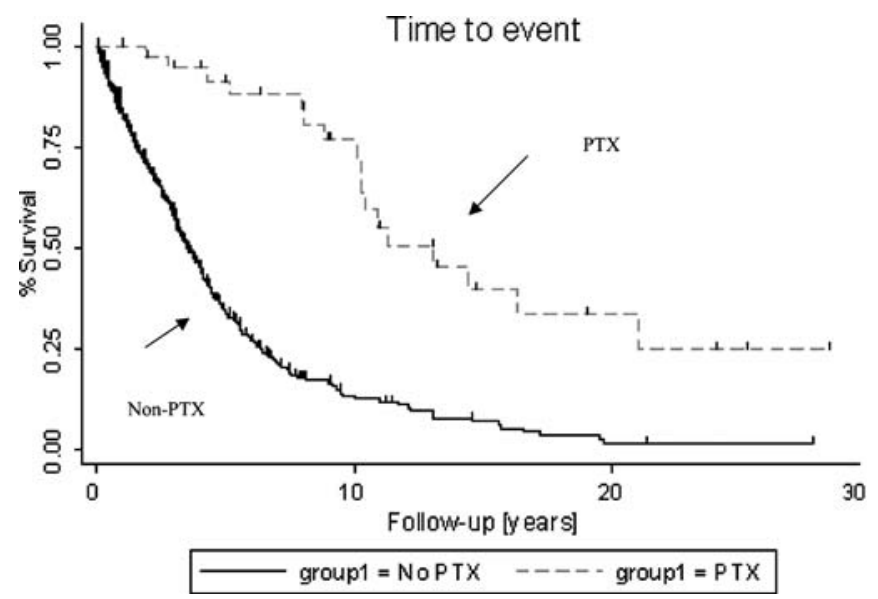

Figure 1. Survival after first dialysis. Kaplan-Meier survival estimates for 40 PTX patients and 664 nonoperated patients with ESRD. Follow-up started on the date of first dialysis, and continued until death or censoring. Censoring took place at survival on 1 March 2003 or first successful kidney transplantation. A kidney transplantation was judged successful if survival of the transplant was greater than one year. 
Table 3.

Patient characteristics (matched case-control study)

\begin{tabular}{|c|c|c|c|c|c|c|c|c|c|}
\hline & \multicolumn{4}{|c|}{$\begin{array}{l}\text { Dialyzed patients with no PTX } \\
\qquad(n=80)\end{array}$} & \multicolumn{4}{|c|}{$\begin{array}{l}\text { Dialyzed patients with PTX } \\
\qquad(n=40)\end{array}$} & \multirow[t]{2}{*}{$P$ Value } \\
\hline & $\mathrm{N}(\%)$ & Mean \pm SD & $\min$ & $\max$ & $\mathrm{N}(\%)$ & Mean \pm SD & $\min$ & $\max$ & \\
\hline Gender $\mathrm{N}$ (\% of male) & $41(51)$ & & & & $18(45)$ & & & & 0.326 \\
\hline \multicolumn{10}{|l|}{ Cause of renal failure } \\
\hline Chronic glomerulonephritis & $26(33)$ & & & & $13(33)$ & & & & 0.579 \\
\hline Polycystic kidney disease & $9(11)$ & & & & $9(23)$ & & & & 0.090 \\
\hline Vascular & $17(21)$ & & & & $4(10)$ & & & & 0.099 \\
\hline Tubulointerstitial & $22(28)$ & & & & $12(30)$ & & & & 0.467 \\
\hline Diabetes & $5(6)$ & & & & $1(3)$ & & & & 0.346 \\
\hline Other or unknown & 7 (9) & & & & $1(3)$ & & & & 0.186 \\
\hline Age at first dialysis & & $55.0 \pm 14.6$ & 26 & 88 & & $42.6 \pm 15.2$ & 14 & 69 & 0.001 \\
\hline Modality of dialysis & & & & & & & & & 0.310 \\
\hline Hemodialysis & $65(81)$ & & & & $29(73)$ & & & & \\
\hline Peritoneal dialysis & $3(4)$ & & & & $4(10)$ & & & & \\
\hline Both modalities & $12(15)$ & & & & $7(18)$ & & & & \\
\hline Time on dialysis (years) & & $8.4 \pm 4.7$ & 0 & 28 & & $9.6 \pm 6.9$ & 1 & 28 & 0.275 \\
\hline Renal graft $(\%)$ & $21(26)$ & & & & $22(55)$ & & & & 0.002 \\
\hline Number of renal transplants (RT) & & & & & & & & & 0.004 \\
\hline Never & $59(74)$ & & & & $18(45)$ & & & & \\
\hline One RT & $17(21)$ & & & & $15(38)$ & & & & \\
\hline Two RT & $4(5)$ & & & & $7(18)$ & & & & \\
\hline Functioning renal graft $(\%)$ & $20(25)$ & & & & $15(38)$ & & & & 0.114 \\
\hline Mean number of cardiovascular risk factors & & $1.4 \pm 1.0$ & 0 & 3 & & $1.2 \pm 0.9$ & 0 & 3 & 0.298 \\
\hline Mean modified Charlson score & & $6.0 \pm 2.6$ & 2 & 16 & & $4.2 \pm 1.6$ & 2 & 13 & 0.001 \\
\hline Charlson score & & & & & & & & & 0.001 \\
\hline Low score $(\leq 3)$ & $14(18)$ & & & & $18(45)$ & & & & \\
\hline Moderate score (4 or 5$)$ & $19(24)$ & & & & $13(33)$ & & & & \\
\hline High score $(6$ or 7$)$ & $27(34)$ & & & & $7(18)$ & & & & \\
\hline Very high score $(\geq 8)$ & $20(25)$ & & & & $2(5)$ & & & & \\
\hline
\end{tabular}

( \pm 10 years), modality of dialysis (hemodialysis, peritoneal dialysis, or both, at any time during the follow-up), modified Charlson score, and parathyroidectomy.

In a univariate model, there was a trend for PTX being associated with prolonged survival (Fig. 2, Table 4). The mortality rate was higher with advanced age at first dialysis and high modified Charlson score. To evaluate to what extent these covariates could affect survival, independent variables were included in a multivariate model. Adjustments for these covariates made the effect of PTX not any more significant (Table 4). The age at first dialysis, the year of first dialysis after 1990, and a highly modified Charlson score were significant and independent predictors of death.

\section{DISCUSSION}

Patients with ESRD are at high risk of cardiovascular morbidity and mortality, particularly from ischemic heart disease and heart failure. ${ }^{19}$ Secondary hyperparathy-

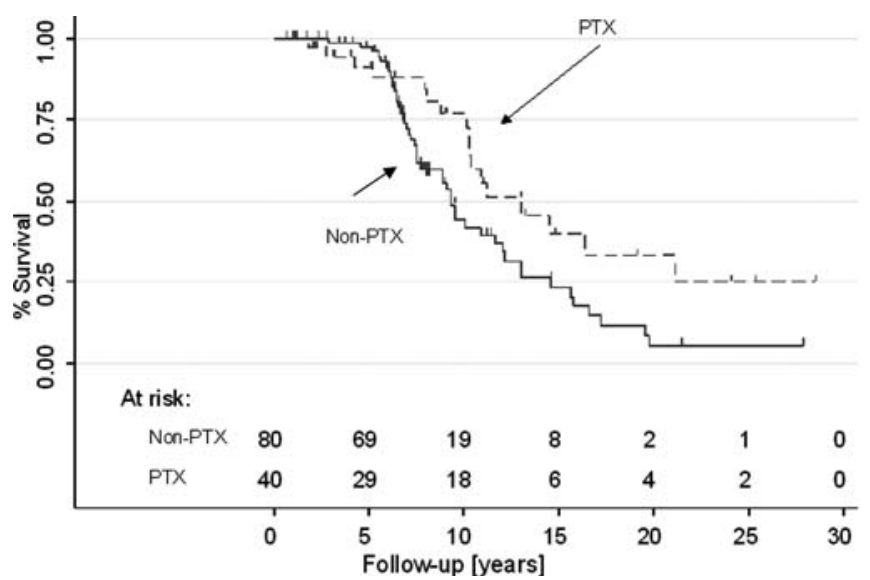

Figure 2. Survival after PTX. We randomly selected two controls for each PTX case who had an equivalent mean duration of dialysis, between the first dialysis and PTX for the PTX group and the beginning of dialysis and any censoring event for the control group (plus or minus 2 years) to analyse the effect of PTX on survival.

roidism is believed to contribute to an increased risk of cardiovascular mortality, but the direct link between PTH and disorders of cardiovascular structure and function in 
Table 4.

Factors influencing mortality rate in patients with end-stage renal disease

\begin{tabular}{|c|c|c|c|c|c|c|}
\hline & Unadjusted OR $(95 \% \mathrm{Cl})$ & $\mathrm{p}$ & $\mathrm{R}^{2}$ & Adjusted OR $(95 \% \mathrm{Cl})$ & $\mathrm{p}$ & $\mathrm{R}^{2}$ \\
\hline PTX & $0.54(0.24-1.17)$ & 0.117 & 0.017 & $1.50(0.49-4.61)$ & 0.478 & \\
\hline Mean $\mathrm{N}$ of cardiovascular risk factors & $0.95(0.64-1.41)$ & 0.789 & 0.001 & $1.06(0.60-1.88)$ & 0.845 & \\
\hline Gender & $1.15(0.55-2.40)$ & 0.704 & 0.001 & $.869(0.30-2.53)$ & 0.797 & \\
\hline Age at first dialysis & $1.08(1.05-1.12)$ & 0.001 & 0.208 & $1.09(1.03-1.15)$ & 0.003 & \\
\hline $\begin{array}{l}\text { Modality of dialysis } \\
\text { Hemodialysis }\end{array}$ & & 0.5244 & 0.009 & & & \\
\hline Peritoneal dialysis & $2.19(0.41-11.51)$ & 0.357 & & $2.50(0.24-26.31)$ & 0.445 & \\
\hline Both modalities & $1.48(0.53-4.13)$ & 0.453 & & $1.79(0.40-7.96)$ & 0.447 & \\
\hline $\begin{array}{l}\text { Charlson score } \\
\text { Low score }(\leq 3)\end{array}$ & & 0.001 & 0.228 & & & \\
\hline Moderate score (4 or 5$)$ & $2.03(0.67-6.18)$ & 0.213 & & $0.863(0.20-3.74)$ & 0.844 & \\
\hline High score $(6$ or 7$)$ & $5.03(1.69-14.98)$ & 0.004 & & $1.37(0.23-8.11)$ & 0.731 & \\
\hline Very high score $(\geq 8)$ & $62.66(7.12-551.47)$ & 0.001 & & $44.50(2.44-811.22)$ & 0.010 & \\
\hline $\begin{array}{l}\text { Years of first dialysis } \\
1970\end{array}$ & & 0.480 & 0.001 & & & \\
\hline 1980 & $1.42(0.56-3.58)$ & 0.457 & & $0.80(0.23-2.76)$ & 0.730 & \\
\hline 1990 & $0.827(0.30-2.24)$ & 0.709 & & $0.06(0.01-0.38)$ & 0.003 & \\
\hline
\end{tabular}

*Whole model.

ESRD is not fully understood. The deleterious effects of high PTH levels in ESRD might be related to an increase in cell calcium content. Previous studies have suggested that high levels of PTH are associated with impaired ventricular diastolic and systolic function. ${ }^{20-24}$ Secondary hyperparathyroidism is also associated with left ventricular hypertrophy. ${ }^{25-29}$ In combination with the higher prevalence of hypertension, this could contribute to the greater prevalence of congestive heart failure in SHPT patients. Several studies have suggested that hyperlipemia in renal failure is related to elevated PTH levels. ${ }^{30-32}$ Decreased PTH-related insulin sensitivity may also augment atherosclerotic changes. ${ }^{33}$ Myocardial disease, along with atherosclerosis of the coronary arteries, ${ }^{34}$ may increase the risk of myocardial infarction and arrhythmias, which could contribute to sudden death, and thus to higher mortality rates among ESRD patients.

Whether PTX reduces the risk of premature death of patients with ESRD and severe SHPT is not clearly accepted because data on the influence of PTX in ESRD on survival are very scarce. A beneficial effect was suggested in a recently published report. ${ }^{15}$ Long-term postPTX survival improved, with a relative hazard ratio for mortality of $0.85(95 \% \mathrm{Cl}: 0.78-0.92)$ after one year. A higher short-term mortality rate was also indicated in the first 30 days after surgery.

It has been demonstrated that PTX leads to a statistically and clinically significant decrease in blood pressure levels. ${ }^{35-37}$ However, these data were not confirmed by others groups. ${ }^{38,39}$ Another recent study has demon- strated that left ventricular function in patients with advanced SHPT and dilated cardiomyopathy markedly improved following PTX. ${ }^{40}$ Goldsmith et al. noted reduced progression of calcification in haemodialysis patients who underwent PTX. ${ }^{41}$

Our own study suggested that the survival rate of patients who develop severe SHPT leading to PTX may differ from that of nonoperated subjects. Indeed, in a first step, we analyzed survival after the first dialysis of all the patients in the study. Mortality was significantly lower in the PTX group, but the patient characteristics were markedly different. We then selected a control group that was more comparable to the PTX patients. The only differences that persisted after matching were age at first dialysis and the modified Charlson morbidity score. Parathyroidectomy patients were younger at first dialysis and had fewer comorbidities. The small number of patients and adjustments for these covariates made the effect of PTX nonsignificant.

It is unlikely that the population had a selection bias, as all cases of SHPT in Geneva are managed at our institution. Thus, patients selected for surgery are more likely to have had advanced disease with symptoms than those selected for conservative management, most of whom had asymptomatic disease with a lower risk prevalence of comorbidity. Nevertheless, some patients may have been in too poor a condition to tolerate surgery, and this would mean that the risk of death for some of those who were managed conservatively might have been higher than for those undergoing surgery. 
The low number of patients and deaths could have limited our ability to detect an effect on survival. This was compensated by the fact that a homogeneous population was studied, with consistent and homogeneous data collection. Finally, the study was performed during the period (1977 to 2002) before new, less-hypercalcemic active vitamin $D$ analogs (such as paricalcitol) and sevelamer became available. It is therefore improbable that survival was altered by modification of treatment strategies. These results could also suggest that PTX should be performed earlier, before these comorbidities develop. But, as serum PTH levels were not analyzed, this assumption remains hypothetical.

In conclusion, we found that the risk of death of patients who developed severe SHPT leading to PTX was different from that of nonoperated subjects. But this apparent difference may be related to the number and severity of associated comorbidities. Thus, ESRD patients who undergo PTX may represent a subset of possibly healthier subjects than those not undergoing PTX.

\section{ACKNOWLEDGMENTS}

The authors acknowledge the editorial assistance of Mrs Marianne Perez and Mrs Praveen Bhalla.

\section{REFERENCES}

1. Slatopolsky E, Gonzalez E, Martin K. Pathogenesis and treatment of renal osteodystrophy. Blood Purif 2003;21:318-326.

2. Gabay $C$, Ruedin $P$, Slosman $D$, et al. Bone mineral density in patients with end-stage renal failure. Am J Nephrol 1993;13:115-123.

3. Block GA. Association of serum phosphorus and calcium $\times$ phosphate product with mortality risk in chronic hemodialysis patients: national study. Am J Kidney Dis 1998;31:607-617.

4. Ganesh SK, Stack AG, Levin NW, et al. Association of elevated serum $\mathrm{PO}(4), \mathrm{Ca} \times \mathrm{PO}(4)$ product, and parathyroid hormone with cardiac mortality risk in chronic hemodialysis patients. J Am Soc Nephrol 2001;12:2131-2138.

5. Chertow GM, Normand SL, Silva LR, et al. Survival after acute myocardial infarction in patients with end-stage renal disease: results from the cooperative cardiovascular project. Am J Kidney Dis 2000;35:1044-1051.

6. Levin A, Djurdjev O, Barrett B, et al. Cardiovascular disease in patients with chronic kidney disease: getting to the heart of the matter. Am J Kidney Dis 2001;38:1398-1407.
7. Collins AJ, Li S, Gilbertson DT, et al. Chronic kidney disease and cardiovascular disease in the Medicare population. Kidney Int Suppl 2003:S24-S31.

8. Marco MP, Craver L, Betriu A, et al. Higher impact of mineral metabolism on cardiovascular mortality in a European hemodialysis population. Kidney Int 2003;63(Suppl. 85):S111-S114.

9. Block GA, Klassen PS, Lazarus JM, et al. mineral metabolism, mortality, and morbidity in maintenance hemodialysis. J Am Soc Nephrol 2004;15:2208-2218.

10. Rix $M$, Andreassen $H$, Eskildsen $P$, et al. Bone mineral density and biochemical markers of bone turnover in patients with predialysis chronic renal failure. Kidney Int 1999;56:1084-1093.

11. Ruedin $\mathrm{P}$, Rizzoli R, Slosman $\mathrm{D}$, et al. Effects of oral calcitriol on bone mineral density in patients with end-stage renal failure. Kidney Int 1994;45:245-252.

12. Frazao JM, Martins $P$, Coburn JW. The calcimimetic agents: perspectives for treatment. Kidney Int 2002;61(Suppl 80):149-154.

13. Cohen EP, Moulder JE. Parathyroidectomy in chronic renal failure: has medical care reduced the need for surgery? Nephron 2001;89:271-273.

14. Kestenbaum B, Seliger SL, Gillen DL, et al. Parathyroidectomy rates among United States dialysis patients: 19901999. Kidney Int 2004;65:282-288.

15. Kestenbaum B, Andress DL, Schwartz SM, et al. Survival following parathyroidectomy among United States dialysis patients. Kidney Int 2004;66:2010-2016.

16. Beddhu S, Bruns FJ, Saul M, et al. A simple comorbidity scale predicts clinical outcomes and costs in dialysis patients. Am J Med 2000;108:609-613.

17. Kaplan EL, Meier P. Nonparametric estimation from incomplete observations. J Am Stat Assoc 1958;53:457-481.

18. Collett D. (1995) Modeling survival data in medical research. In: Zidek JV (ed). Tests in Statistical Science Chapman and Hall, London, pp 40-43.

19. Parfrey PS, Harnett JD, Barre PE. The natural history of myocardial disease in dialysis patients. J Am Soc Nephrol 1991;2:2-12.

20. Rostand SG, Sanders C, Kirk KA, et al. Myocardial calcification and cardiac dysfunction in chronic renal failure. Am J Med 1988;85:651-657.

21. Rostand SG, Sanders C, Rutsky EA. Cardiac calcification in uremia. Contrib Nephrol 1993;106:26-29.

22. Coratelli $\mathrm{P}$, Buongiorno E, Petrarulo $\mathrm{F}$, et al. Pathogenetic aspects of uremic cardiomyopathy. Miner Electrolyte Metab 1989;15:246-253.

23. Ohara N, Hiramatsu K, Shigematsu S, et al. Effect of parathyroid hormone on left ventricular diastolic function in patients with primary hyperparathyroidism. Miner Electrolyte Metab 1995;21:63-66.

24. Shane E, Mancini D, Aaronson K, et al. Bone mass, vitamin $\mathrm{D}$ deficiency, and hyperparathyroidism in congestive heart failure. Am J Med 1997;103:197-207. 
25. Harnett JD, Parfrey PS, Griffiths SM, et al. Left ventricular hypertrophy in end-stage renal disease. Nephron 1988;48:107-115.

26. Duprez D, Bauwens F, De Buyzere M, et al. Relationship between parathyroid hormone and left ventricular mass in moderate essential hypertension. J Hypertens Suppl 1991;9:S116-S117.

27. London GM, De Vernejoul MC, Fabiani F, et al. Secondary hyperparathyroidism and cardiac hypertrophy in hemodialysis patients. Kidney Int 1987;32:900-907.

28. Symons C, Fortune F, Greenbaum RA, et al. Cardiac hypertrophy, hypertrophic cardiomyopathy, and hyperparathyroidism—an association. Br Heart J 1985;54:539542.

29. Saleh FN, Schirmer H, Sundsfjord J, et al. Parathyroid hormone and left ventricular hypertrophy. Eur Heart $\mathrm{J}$ 2003;24:2054-2060.

30. Lacour B, Roullet JB, Liagre AM, et al. Serum lipoprotein disturbances in primary and secondary hyperparathyroidism and effects of parathyroidectomy. Am J Kidney Dis 1986;8:422-429.

31. Akmal M, Kasim SE, Soliman AR, et al. Excess parathyroid hormone adversely affects lipid metabolism in chronic renal failure. Kidney Int 1990;37:854-858.

32. Liang K, Oveisi F, Vaziri ND. Role of secondary hyperparathyroidism in the genesis of hypertriglyceridemia and VLDL receptor deficiency in chronic renal failure. Kidney Int 1998;53:626-630.

33. Defronzo RA, Alvestrand $A$, Smith $D$, et al. Insulin resistance in uremia. J Clin Invest 1981;67:563-568.
34. Roberts WC, Waller BF. Effect of chronic hypercalcemia on the heart. An analysis of 18 necropsy patients. Am J Med 1981;71:371-384.

35. Coen G, Calabria S, Bellinghieri G, et al. Parathyroidectomy in chronic renal failure: short- and long-term results on parathyroid function, blood pressure and anemia. Nephron 2001;88:149-155.

36. Pizzarelli F, Fabrizi F, Postorino M, et al. Parathyroidectomy and blood pressure in hemodialysis patients. Nephron 1993;63:384-389.

37. Goldsmith DJ, Covic AA, Venning MC, et al. Blood pressure reduction after parathyroidectomy for secondary hyperparathyroidism: further evidence implicating calcium homeostasis in blood pressure regulation. Am J Kidney Dis 1996;27:819-825.

38. Zucchelli $P$, Santoro A, Zucchelli A, et al. Long-term effects of parathyroidectomy on cardiac and autonomic nervous system functions in haemodialysis patients. Nephrol Dial Transplant 1988;3:45-50.

39. Ifudu O, Matthew JJ, Macey LJ, et al. Parathyroidectomy does not correct hypertension in patients on maintenance hemodialysis. Am J Nephrol 1988;18:28-34.

40. Goto $\mathrm{N}$, Tominaga $\mathrm{Y}$, Ueki $\mathrm{T}$, et al. Left ventricular function of dilated cardiomyopathy in patients with advanced secondary hyperparathyroidism was dramatically improved by parathyroidectomy. J Am Soc Nephrol 2003;14:683A.

41. Goldsmith DJ, Covic AA, Sambrook PA, et al. Vascular calcification in long-term hemodialysis patients in single unit: a retrospective analysis. Nephron 1997;77:37-43. 